\title{
Nanometer-Resolved Collective Micromeniscus Oscillations through Optical Diffraction
}

\author{
Helmut Rathgen, ${ }^{1,2, *}$ Kazuyasu Sugiyama, ${ }^{3,2}$ Claus-Dieter Ohl, ${ }^{3,2,4}$ Detlef Lohse, ${ }^{3,2}$ and Frieder Mugele ${ }^{1,2}$ \\ ${ }^{1}$ Physics of Complex Fluids, University of Twente, The Netherlands \\ ${ }^{2}$ J. M. Burgers Centre of Fluid Dynamics and MESA ${ }^{+}$- and IMPACT-Institutes, University of Twente, The Netherlands \\ ${ }^{3}$ Physics of Fluids, University of Twente, The Netherlands \\ ${ }^{4}$ School of Physical and Mathematical Sciences, Nanyang Technological University, Singapore
}

(Received 16 July 2007; published 21 November 2007)

\begin{abstract}
We study the dynamics of periodic arrays of micrometer-sized liquid-gas menisci formed at superhydrophobic surfaces immersed into water. By measuring the intensity of optical diffraction peaks in real time, we are able to resolve nanometer-scale oscillations of the menisci with submicrosecond time resolution. Upon driving the system with an ultrasound field at variable frequency, we observe a pronounced resonance at a few hundred kilohertz, depending on the exact geometry. By modeling the system using the unsteady Stokes equation, we find that this low resonance frequency is caused by a collective mode of the acoustically coupled oscillating menisci.
\end{abstract}

DOI: 10.1103/PhysRevLett.99.214501

PACS numbers: 47.55.dd, 42.25.Fx, 43.35.+d

Superhydrophobic surfaces have attracted much theoretical and experimental interest [1]. The entrapment of gas at the textured surface reduces the actual interfacial area between the solid and the liquid, which is at the origin of the distinctive properties of these materials, including the large contact angle, the low contact angle hysteresis [2], the self-cleaning effect [3], and the large hydrodynamic slip [4]. The superhydrophobic state is closely related to the shape of the microscopic liquid-gas interfaces spanning between the ridges of the texture [5]. In this Letter, we characterize the dynamics of these micromenisci and introduce an optical diffraction measurement that reveals their nanoscale motion. We measure in real time the optical diffraction intensity from a periodic array of micromenisci. By driving the system with an ultrasound field at variable frequency, we measure its frequency response, and we identify a well-defined resonance peak with a center frequency well below the expectations for a single micromeniscus. By modeling the system using the unsteady Stokes equation and monopole interaction, we show that this frequency reduction is due to acoustic coupling between menisci.

Figure 1 shows the geometry of our system. It consists of $1 \times 1 \mathrm{~mm}^{2}$ wide hexagonal and square arrays of micrometer-sized cylindrical holes (radii $R=2 \mu \mathrm{m}$ or $3 \mu \mathrm{m}$, depth $H=15 \mu \mathrm{m}$, nearest neighbor distance $a=$ 15 or $25 \mu \mathrm{m})$. The samples were fabricated from Si (110) using standard microlithography. Subsequently, the surfaces were hydrophobized by vapor deposition of a monolayer of $1-\mathrm{H}, 1-\mathrm{H}, 2-\mathrm{H}, 2 \mathrm{H}$-perfluorodecyltrichlorosilane, following Ref. [6]. The advancing and receding contact angles on an unstructured surface are $\gamma_{a}=116^{\circ}$ and $\gamma_{r}=$ $104^{\circ}$, respectively. Upon immersing the samples into demineralized water, ambient air is entrapped in every hole, leaving a water-air meniscus behind that is pinned at the ridge of the hole. Owing to the hydrostatic pressure, the menisci are bent inwards with equilibrium curvature $\kappa_{0}=$ $\rho g h / \sigma$ (as we have also checked by independent measurement), where $g$ is the acceleration of gravity, $\sigma$ is the water surface tension, and $h \approx 0.1 \mathrm{~m}$ is the distance between the sample and the free water surface. This implies that the system is in diffusion equilibrium and the gas pressure in the hole is the ambient pressure. An Ar-ion laser $(\lambda=$ $488 \mathrm{~nm}, s$-polarized) is used to illuminate the sample under an angle typically between $60^{\circ}$ and $70^{\circ}$ with respect to normal incidence (see Fig. 2). The diffracted intensity is measured with a photodiode positioned at a selected diffraction peak, typically chosen in the vicinity of the specular reflected beam. A broadband piezoelectric ultrasound transducer is placed at its focal distance from the sample. The ultrasound transducer is excited to emit finite wave trains by using an arbitrary function generator. The ultrasound pressure at the sample is of the order $10^{2}-10^{3} \mathrm{~Pa}$, which is small compared to a critical static pressure above which filling occurs: $P_{c}=2 \sigma \cos \left(\gamma_{a}\right) / R \approx 2.1 \times 10^{4} \mathrm{~Pa}$. To check the dynamic stability of the menisci, we increased the ultrasound pressure to much larger values and observed how the intensity oscillations disappeared at a defined threshold. The ultrasound pressure is kept constant during a frequency sweep by controlling the driving voltage according to the transducer frequency re- a)

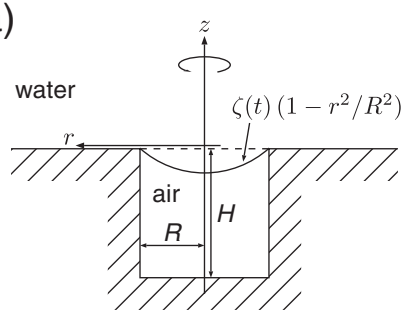

b)

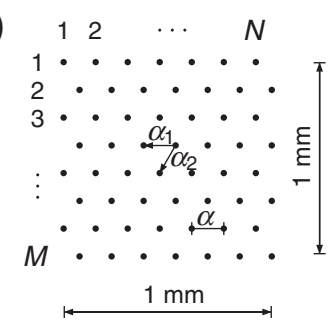

FIG. 1. (a) Schematic figure of a single micromeniscus. (b) Pattern of an array of micromenisci. Arrows denote the primitive translations and nearest neighbor distance $a$. 
a) Setup

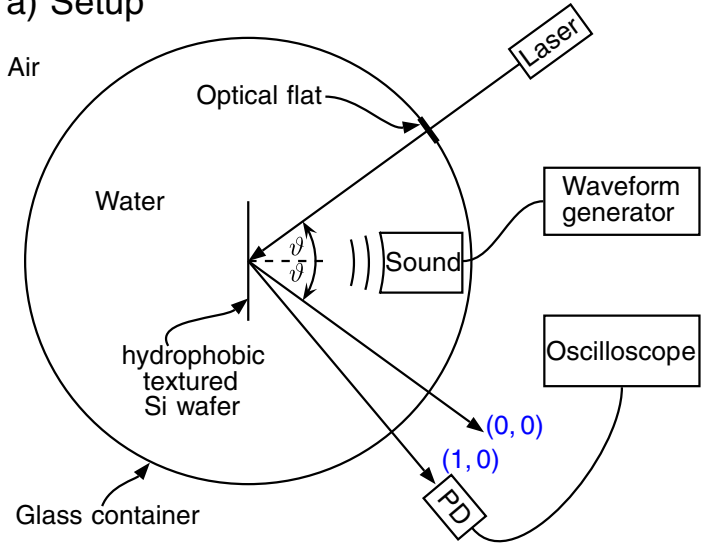

b) Diffraction pattern at $60^{\circ}$

c) Ultrasound response

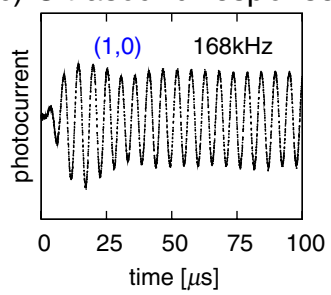

FIG. 2 (color online). (a) Schematic figure of the experiment. (b) Inverted photograph of the diffraction pattern $\left(\vartheta \approx 60^{\circ}\right)$. Numbers indicate Miller indices of diffraction orders. (c) ac component of the light intensity measured in the $(1,0)$ diffraction order at the beginning of an ultrasound wave train.

sponse. Figure 2(c) shows typical raw data corresponding to the beginning of a wave train. After a transient lasting for a few oscillation cycles, the signal becomes sinusoidal with a constant amplitude. This amplitude is extracted from the raw data by calculating the root mean square.

The diffracted intensity depends in a highly nonlinear way on the deflection of the menisci. To assure a linear relation between the actual deflection and the measured intensity, the menisci oscillations have to be small. This can be seen in Fig. 3(a). While the diffracted intensity follows the sinusoidal driving pressure at low driving amplitudes (solid line), it is distorted at larger driving amplitudes (dashed line). Prior to an experiment, we reduce the ultrasound pressure until the undistorted sinus is observed.

To find the magnitude of the corresponding meniscus deflections, we consider the optical diffraction of the sample. In the Fraunhofer limit, the diffracted intensity is proportional to the intensity scattered by a single unit cell, and, for each unit cell, the scattered intensity is governed by the interference of the elementary waves emitted from the cell volume [7]. In our case, the incident angle is large, and no light reaches the bottom of the holes such that the interference takes place between the waves emitted from the plane silicon surface and the waves emitted from the meniscus. Qualitatively, as the meniscus is deflected-consider the position $\zeta$ of the apex of the meniscus - the intensity of a diffraction order (with dif- a)

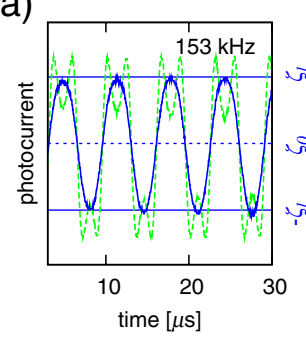

b)

$\zeta[\mu \mathrm{m}]$

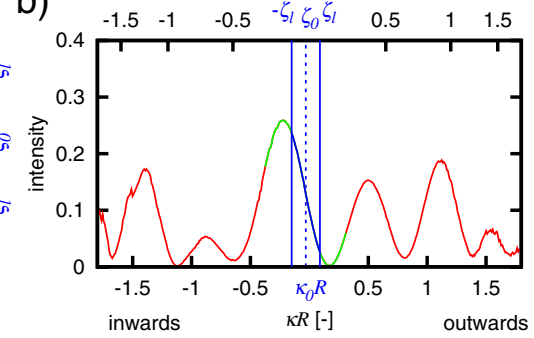

FIG. 3 (color online). (a) Time-resolved intensity of the $(1,0)$ diffraction order, corresponding to small ultrasound pressure $(\Delta P \approx 400 \mathrm{~Pa}$ ) (solid line) and large ultrasound pressure $(\Delta P \approx$ $800 \mathrm{~Pa}$ ) (dashed line). (b) Calculated intensity of the first diffraction order as a function of meniscus deflection for a rectangular surface profile with groove width $w=2 R\left(\vartheta=66^{\circ}\right)$. The data are displayed simultaneously in terms of the displacement $\zeta$ and the nondimensionalized curvature $\kappa R$. Both are related geometrically by $\zeta=2 / \kappa\left(1-\sqrt{1-R^{2} \kappa^{2} / 4}\right)$. The equilibrium curvature $\kappa_{0} R=0.04$ corresponds to $\zeta_{0}=30 \mathrm{~nm}$.

fraction angle $\approx$ incident angle) changes sinusoidally with a period $T=\lambda /[2 n \cos (\vartheta)]$, where $n=1.33$ is the refractive index of water. To analyze these simple observations in detail, we performed a diffractive optics calculation using the multilayer rigorous coupled wave analysis in the formulation of Ref. [8]. This method allows for calculating an exact solution to the Maxwell equations for the optical response of arbitrary periodic surface profiles. In Fig. 3(b), we show the resulting diffraction intensity as a function of the meniscus deflection. The typical distance between two adjacent peaks corresponds to the period evaluated from the simple Fraunhofer arguments above. The result shows that the diffracted intensity is indeed linear in the meniscus deflection in a range $\left[-\zeta_{l},+\zeta_{l}\right]$ around the meniscus equilibrium position $\zeta_{0}$, and we find $\zeta_{l} \approx 90 \mathrm{~nm}$. Note that the extent of the linear range depends on the incident angle $\vartheta$. It is larger for larger incident angles, as can be seen readily from the simple expression for $T$. Thus, the large angles that are used in the experiment allow for large meniscus oscillations. For incident angles above the angle of total reflection between water and air $\left(48.6^{\circ}\right)$, in addition the relative contribution of the menisci to the scattered intensity is large.

The theoretical result for the linear range is the key to converting the measured intensity variations into absolute meniscus deflections. Since we have to assure linearity between intensity and meniscus deflection at all ultrasound frequencies, the peak of the resonance curve shown in the following has the height $\zeta_{l}$. The slope of the linear range together with the relative noise of the photodiode determines the resolution of the deflection measurement. It is of the order $1 \mathrm{~nm}$ under the given conditions.

Figure 4 shows a typical measured frequency response. The sample displays a single resonance at $f_{r}=153 \pm$ $5 \mathrm{kHz}$. Similar curves were obtained for all samples. Table I shows that the observed resonance frequency in- 


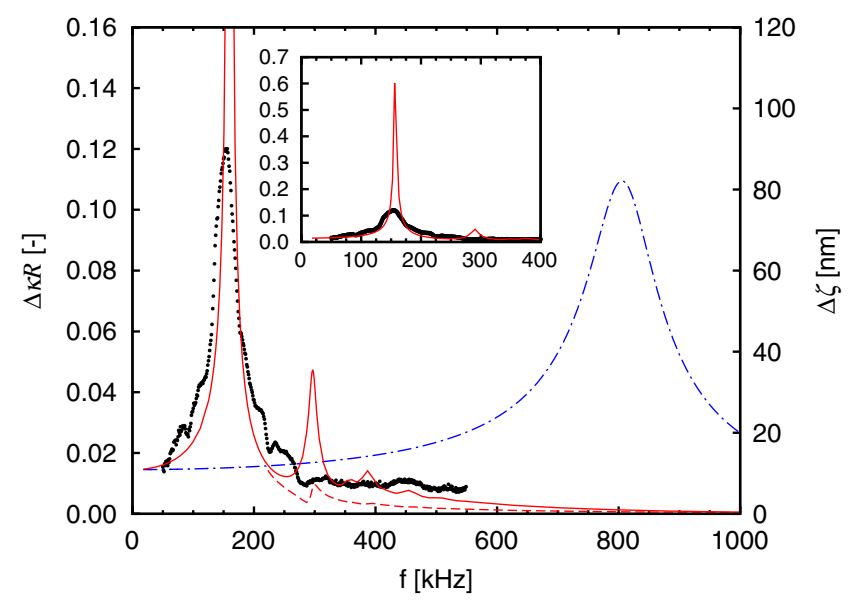

FIG. 4 (color online). Frequency response of an array of micromenisci with a hexagonal pattern, $a=15 \mu \mathrm{m}$, and $R=$ $3 \mu \mathrm{m}$. Crosses show experimental data. The dashed-dotted line shows the theory for a single meniscus. Theoretical data for the array are displayed in terms of the mean (solid line) and the root mean square deflection (dashed line).

creases both with increasing nearest neighbor distance and with decreasing hole size.

To understand the observations quantitatively, we consider first the response of a single meniscus under the influence of the pressure field $P(t)=P_{0}+\Delta P e^{i 2 \pi f t}$, where $f$ is the ultrasound frequency, $P_{0}$ is the ambient pressure, and $\Delta P$ is the amplitude of the ultrasound pressure. Since $\zeta_{0} \ll \Delta \zeta, R$, as follows with the values evaluated above, we approximate the meniscus as flat in equilibrium and its deflections as parabolic $\zeta(r)=\zeta(1-$ $\left.r^{2} / R^{2}\right)$. The parabolic shape implies that the curvature is uniform up to $O\left(\zeta^{3}\right)$ giving right to the Laplace law. As described in Ref. [9], the smallness of the deflections $\Delta \zeta \ll R$, and the high frequency $f \gg \nu / R^{2}$ of the oscillations ( $\nu$ kinematic viscosity), allow us to omit the nonlinear term in the Navier-Stokes equation, and hence the dynamics of the system is governed by the unsteady Stokes equation. The system can then be described as a harmonic oscillator

$$
\left\{\Phi\left(\omega^{*}\right)+i \Psi\left(\omega^{*}\right)+K\right\} \Delta \zeta^{*}=-\Delta P^{*} .
$$

The transfer functions $\Phi$ and $\Psi$ account for the inertia and the viscous damping, respectively, due to the oscillatory flow fields. All quantities are nondimenionalized $\omega^{*}=$ $2 \pi f R^{2} / \nu, \Delta \zeta^{*}=\zeta / R$, and $\Delta P^{*}=\Delta P R^{2} /(\rho \nu)$. Since polytropic and thermal dissipative effects [10] can be neglected, the potential term reduces to a dimensionless spring constant $K=R /\left(\rho \nu^{2}\right)\left[P_{0} R^{2} /(2 H)+4 \sigma\right]$. The first term with the ambient pressure is due to the isothermal compression of the gas and is negligible throughout this work, and the second term with the surface tension $\sigma$ is due to the surface energy of the liquid-gas interface. The computation of $\Phi$ and $\Psi$ is performed by solving the unsteady Stokes equation in cylindrical coordinates with classical no-slip and free slip boundary conditions at the
TABLE I. Resonance frequencies of the lowest collective mode $f_{c}$ as a function of lattice constant, pattern geometry, and menisci radius. $H$ and $S$ denote the hexagonal and the square pattern, respectively. $f_{s}$ denotes the resonance frequency of a corresponding single meniscus, $f_{r}$ the experimental result.

\begin{tabular}{cccccccc}
\hline \hline$R(\mu \mathrm{m})$ & $a(\mu \mathrm{m})$ & Pattern & $N$ & $M$ & $f_{s}(\mathrm{kHz})$ & $f_{c}(\mathrm{kHz})$ & $f_{r}(\mathrm{kHz})$ \\
\hline 3 & 15 & $H$ & 66 & 76 & 805 & 159 & $153 \pm 5$ \\
3 & 15 & $S$ & 67 & 67 & 805 & 170 & $177 \pm 5$ \\
3 & 25 & $H$ & 40 & 46 & 805 & 258 & $230 \pm 20$ \\
3 & 25 & $S$ & 41 & 41 & 805 & 275 & $240 \pm 20$ \\
2 & 15 & $H$ & 66 & 76 & 1433 & 346 & $285 \pm 20$ \\
2 & 15 & $S$ & 67 & 67 & 1433 & 368 & $290 \pm 20$ \\
2 & 25 & $H$ & 40 & 46 & 1433 & 549 & $400 \pm 20$ \\
2 & 25 & $S$ & 41 & 41 & 1433 & 584 & $410 \pm 20$ \\
\hline \hline
\end{tabular}

solid-liquid and the liquid-gas interface, respectively. A detailed account of the calculations will be given elsewhere [11]. While analytical expressions can be found for both the high and low frequency limits, $\Phi$ and $\Psi$ have to be computed numerically in the intermediate frequency range $10^{0}<\omega^{*}<10^{2}$, which is relevant for the present experiments. The dashed-dotted line in Fig. 4 shows the solution for a $R=3 \mu \mathrm{m}$ hole with physical parameters of water $\rho=10^{3} \mathrm{~kg} / \mathrm{m}^{3}, \nu=10^{-6} \mathrm{~m}^{2} / \mathrm{s}, \quad \sigma=73 \times 10^{-3} \mathrm{~N} / \mathrm{m}$, and ultrasound pressure $\Delta P=390 \mathrm{~Pa}$ as used in the respective experiment. The obtained resonance frequency is approximately 5 times larger than the one observed experimentally. Clearly, the single meniscus theory fails to describe the dynamics of the system.

To resolve the discrepancy, we consider the dynamic coupling between the menisci. As the menisci oscillate in the external pressure field, they emit pressure waves that affect the other menisci. Denoting the nondimensionalized distance between the $i$ th and the $j$ th menisci by $d_{i j}^{*}=$ $d_{i j} / R$, the additional force acting on the $i$ th meniscus can be expressed in terms of a multipole expansion $-\Delta \zeta_{j}^{*} \omega^{* 2} /\left(4 d_{i j}^{*}\right)+O\left(d_{i j}^{*-2}\right)$, where $\Delta \zeta_{j}^{*}$ is the deflection amplitude of the $j$ th meniscus (see, e.g., $[12,13]$ ). To analyze the dynamics of the entire meniscus array, we extend the equation of motion of the single meniscus [Eq. (1)] by the additional forces generated by all other menisci, keeping only the monopole term. We arrive at the coupled equations of motion

$$
\left\{\Phi\left(\omega^{*}\right)+i \Psi\left(\omega^{*}\right)+K\right\} \Delta \zeta_{i}^{*}=-\Delta P^{*}+\sum_{i \neq j}^{N \cdot M} \frac{\omega^{* 2}}{4 d_{i j}^{*}} \Delta \zeta_{j}^{*}
$$

The coupling term gives rise to an additional effective mass, which reduces the resonance frequency, as required. We solve Eq. (2) for the individual deflection amplitudes $\Delta \zeta_{i}^{*}$ by numerical matrix inversion. From the result, we evaluate the mean $\left\langle\Delta \zeta^{*}\right\rangle=\left|\sum_{i=1}^{\mathrm{NM}} \Delta \zeta_{i}^{*}\right| / \mathrm{NM}$ and the root mean square $\left\langle\Delta \zeta^{* 2}\right\rangle^{1 / 2}=\left(\sum_{i=1}^{\mathrm{NM}}\left|\Delta \zeta_{i}^{*}\right|^{2}\right)^{1 / 2} / \mathrm{NM}$ deflection amplitude (see discussion below). The results are shown in 
a)
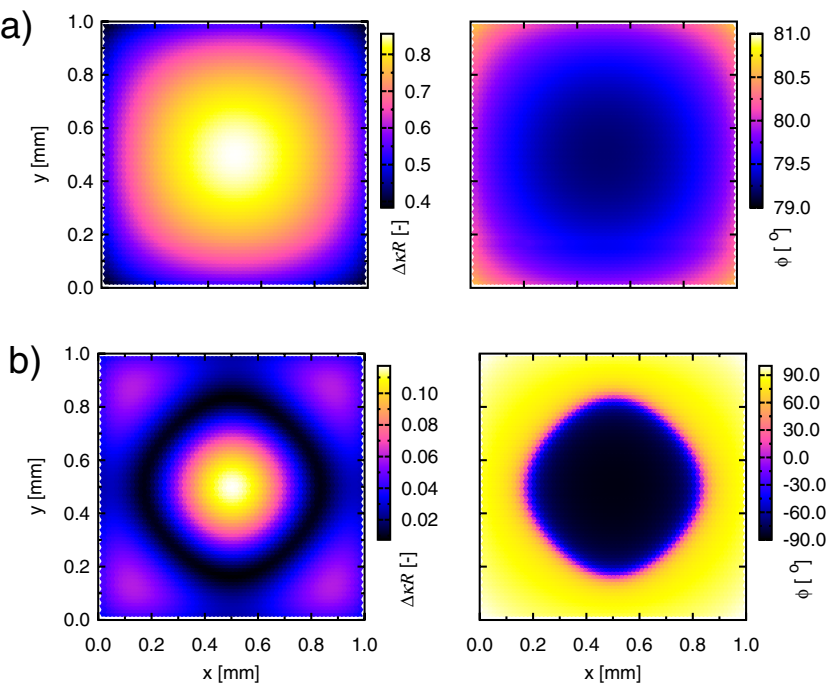

FIG. 5 (color online). Amplitude and phase of an array of micromenisci with a hexagonal pattern, $a=15 \mu \mathrm{m}$, and $R=$ $3 \mu \mathrm{m}$ (a) at the fundamental collective mode $(159 \mathrm{kHz})$ and (b) at the second collective mode $(290 \mathrm{kHz})$. Note that the color scale for the phase is narrower in (a) as compared to (b).

Fig. 4 with the solid and the dashed line, respectively. For the moment note that both curves are nearly identical up to frequencies well above the lowest resonance. The theoretically obtained resonance frequency $f_{c}=159 \mathrm{kHz}$ is in excellent agreement with the experimental data, showing that collective effects are crucial for the dynamics of the system. Moreover, the good agreement shows that monopole interaction is effective beyond its obvious range of applicability where $a \gg R$.

As can be seen in the inset in Fig. 4, the theory overestimates the amplitude of the resonance which is presumably due to the neglect of the bulk dissipation that arises in the collective flow field. Second, and more interestingly, the theoretical data for the root mean square display a second resonance at $290 \mathrm{kHz}$ which is absent in the experimental data. To understand the latter effect, we plot the calculated amplitude and phase for the two lowest resonances as a function of the meniscus position within the array in Fig. 5. For the lowest resonance, all menisci oscillate essentially in phase, whereas at the second resonance, the menisci in the center of the array and the ones along the edge oscillate $180^{\circ}$ out of phase and the amplitude displays a node at the ring-shaped boundary between the two regions. To evaluate the diffraction intensity for such arrays of nonidentical scatterers, we note that the variation of the meniscus deflection gives rise to phase differences between the waves emitted from different unit cells that are much smaller than $2 \pi$-owing to the particular experimental condition assuring that the diffracted intensity is linear in the meniscus displacement. By extending the above Fraunhofer picture, one shows that the diffraction intensity is linear in the individual menisci deflections, and thus the experiment measures the mean deflection. Thus, we have to compare the experiment to the theoretical mean deflection, where the second resonance is indeed nearly invisible. Note that the expression for the mean as given above accounts for the phase since the deflection amplitudes $\Delta \zeta_{i}^{*}$ are complex.

The reduction of the resonance frequency predicted by the theory is confirmed for all surface patterns investigated in the experiment (see Table I). Since the coupling between the menisci is inversely proportional to the distance, the frequency reduction is more pronounced for smaller lattice constants. Similarly, it is more pronounced for the hexagonal lattices than for the square ones, owing to the larger number of nearest neighbors. At this moment, we have no clear explanation for the slight overestimation of the resonance frequency for the samples with smaller radius.

In conclusion, highly mobile micromenisci are present at the textures of superhydrophobic surfaces. Their dynamics are determined by collective modes with resonance frequencies that are much smaller than the resonance frequency of a single isolated meniscus. Superhydrophobic surfaces with particularly large contact angles or large slip length are expected to show the lowest resonance frequencies. Optical diffraction has proven to be an accurate tool for studying superhydrophobic surfaces and their nanoscopic hydrodynamics. It remains a challenge to extend the optical diffraction technique to the accurate study of the microscopic shape of the menisci, presumably by measuring also the angular dependence of the diffraction pattern and subsequently solving the inverse diffraction problem, following upcoming ideas in theoretical diffractive optics.

We gratefully acknowledge stimulating discussions with A. Prosperetti and M. Sbragaglia and experimental support by N. Bremond. This work was supported through the DFG (Grant No. MU 1472/4).

*helmut.rathgen@web.de

[1] D. Quéré, Rep. Prog. Phys. 68, 2495 (2005).

[2] A. Lafuma and D. Quéré, Nat. Mater. 2, 457 (2003).

[3] A. Nakajima et al., Langmuir 16, 7044 (2000).

[4] P. Joseph et al., Phys. Rev. Lett. 97, 156104 (2006).

[5] C. Journet et al., Europhys. Lett. 71, 104 (2005).

[6] T. M. Mayer et al., J. Vac. Sci. Technol. B 18, 2433 (2000).

[7] M. Born and E. Wolf, Principles of Optics (Cambridge University Press, Cambridge, England, 1999), 7th ed.

[8] M. G. Moharam et al., J. Opt. Soc. Am. A 12, 1077 (1995).

[9] L.D. Landau and E. M. Lifshitz, Fluid Mechanics (Pergamon, Oxford, 1959).

[10] X. M. Chen and A. Prosperetti, J. Acoust. Soc. Am. 104, 1389 (1998).

[11] H. Rathgen et al. (to be published).

[12] V. Leroy et al., Eur. Phys. J. E 17, 189 (2005).

[13] N. Bremond et al., Phys. Rev. Lett. 96, 224501 (2006). 